OPEN ACCESS

Edited by:

Zhenbo Xu,

University of Tennessee Health Science Center (UTHSC),

United States

Reviewed by:

Murat Akova,

Hacettepe University, Turkey

Yunsong Yu,

Zhejiang University, China

*Correspondence: Qiwen Yang

yangqiwen81@vip.163.com Yingchun Xu

xycpumch@139.com

Specialty section:

This article was submitted to Antimicrobials, Resistance

and Chemotherapy,

a section of the journal

Frontiers in Microbiology

Received: 27 March 2020

Accepted: 24 July 2020

Published: 04 September 2020

Citation:

Zhang $H$, Zhang $G$, Zhang J,

Duan S, Kang Y, Yang $Q$ and $X u Y$ (2020) Antimicrobial Activity of Colistin Against Contemporary $(2015$ - 2017)

$P$. aeruginosa and $A$. baumanni Isolates From a Chinese Surveillance Program. Front. Microbiol. 11:1966.

doi: 10.3389/fmicb.2020.01966

\section{Antimicrobial Activity of Colistin Against Contemporary (2015 - 2017) $P$. aeruginosa and $A$. baumannii Isolates From a Chinese Surveillance Program}

\author{
Hui Zhang ${ }^{1}$, Ge Zhang1, Jingjia Zhang ${ }^{1}$, Simeng Duan', Yue Kang ${ }^{2}$, Qiwen Yang ${ }^{1 *}$ and \\ Yingchun $\mathrm{Xu}{ }^{1 *}$ \\ ${ }^{1}$ Department of Clinical Laboratory, Peking Union Medical College Hospital, Peking Union Medical College, Chinese \\ Academy of Medical Sciences, Beijing, China, ${ }^{2}$ MRL Global Medical Affairs, MSD China, Shanghai, China
}

Objective: To investigate the incidence and susceptibilities of non-fermenting bacteria isolates from Chinese respiratory (RTI), intra-abdominal (IAI) and urinary tract (UTI) infections to antimicrobial agents between 2015 and 2017.

Methods: In total, 3,246 non-fermentative bacteria were collected from 21 hospitals and 9 hospital departments across 7 regions of China. A central testing laboratory was employed to determine antimicrobial susceptibilities using appropriate standards of interpretation.

Results: The majority of the isolates were Acinetobacter baumannii ( $n=1,360,41.9 \%)$ and Pseudomonas aeruginosa ( $n=1,341,41.3 \%)$. Overall multidrug resistance (MDR) and carbapenem resistance (CR) rates of Acinetobacter baumannii were 80.1 and 78.7\% with MDR and CR rates in RTIs, IAls, and UTIs of 82.0 and $81.0 \%, 82.6$ and $81.0 \%$ as well as 53.1 and $46.9 \%$. Overall MDR and CR rates of Pseudomonas aeruginosa isolates were 36.2 and $38.9 \%$ with 41.8 and $44.3 \%, 29.3$ and $36.1 \%$ as well as 24.2 and $20.2 \%$ MDR and CR rates in RTIs, IAls, and UTIs. Overall susceptibility rates to imipenem, meropenem, amikacin, ciprofloxacin, cefepime and piperacillintazobactam were 21.1, 21.3, 33.0, 18.4, 19.2, and 19.6\% for Acinetobacter baumannil and 56.5, 58.5, 88.4, 63.1, 63.1, and 55.63\% for Pseudomonas aeruginosa isolates, whereas for colistin they were 95.7 and $94.6 \%$, respectively. In all departments and regions of China, susceptibility rates of Pseudomonas aeruginosa and Acinetobacter baumannii isolates to colistin were constantly above $80 \%$.

Conclusion: Due to the high MDR and CR rates for Pseudomonas aeruginosa and Acinetobacter baumannii, isolates obtained from RTIs, IAls, and UTIs only maintained high susceptibility rates to colistin between 2015 and 2017 .

\footnotetext{
Keywords: Pseudomonas aeruginosa, Acinetobacter baumannii, carbapenem, colistin, RTI, IAI, UTI
} 


\section{INTRODUCTION}

Non-fermenting bacteria are a group of aerobic or facultative anaerobic, spore-free, Gram-negative bacteria that do not use glucose or use only oxidized forms; most are conditional pathogens, which mainly cause nosocomial infections, and clinical treatment is very difficult (Mesaros et al., 2007; Perez et al., 2007). In recent years, due to the extensive application of various medical invasive examinations, diagnosis and treatment techniques, as well as broad-spectrum antibacterial drugs, the number of nosocomial infections caused by non-fermentation bacteria has gradually increased, with Acinetobacter baumannii (A. baumannii) and Pseudomonas aeruginosa ( $P$. aeruginosa) being the most common.

Acinetobacter baumannii is an opportunistic pathogen and is one of the most common nosocomial pathogens in clinical practice in China (Chen et al., 2012). Due to the widespread use of antibacterial drugs, especially $\beta$-lactam antibiotics, the isolation rates of A. baumannii in clinical trials are on the rise, and its drug resistance has also shown an upward trend. Indeed, multi-drug resistant and even pan-resistant strains are common (Chopra et al., 2013). A. baumannii often causes respiratory, urinary, skin, soft tissue and bloodstream infections. It is one of the main pathogens causing ventilator-associated pneumonia in the United States and Europe (Fernández-Barat et al., 2017; Koulenti et al., 2017). The pathogenicity and drug resistance mechanism of $P$. aeruginosa have recently been extensively investigated (Dimopoulos et al., 2020). An investigation into healthcare associated infections in the US revealed that $P$. aeruginosa was the 6th most commonly found pathogen in infections as well as the 2nd most common organism causing pneumonia (Magill et al., 2014). Furthermore, this organism has evolved various defenses that render it resistance to various classes of antibiotics including the $\beta$-lactams; $P$. aeruginosa is often cited as being multidrug resistant (MDR) (Castanheira et al., 2014). This has serious consequences as antibiotic therapy to treat MDR $P$. aeruginosa are severely limited, particularly in areas of China where MDR organisms are widely dispersed (Ruiz-Garbajosa and Canton, 2017; El Chakhtoura et al., 2018).

As MDR $P$. aeruginosa and A. baumannii infections are increasing, available antibiotics to treat infections are limited (Karageorgopoulos and Falagas, 2008; Pachon and Vila, 2009; Towner, 2009). Colistin may be one of the most effective antibiotics against $P$. aeruginosa or A. baumannii resistant to carbapenems (Yuan and Tam, 2008), but as a last line of defense the colistin sensitivities of $P$. aeruginosa and A. baumannii is a major concern. The Study for Monitoring Antimicrobial Resistance Trends (SMART) is a global surveillance project designed to collect and monitor in vitro antimicrobial susceptibilities of isolates obtained from patients with intra-abdominal infections (IAIs), respiratory tract infection (RTIs) and urinary tract infections (UTIs).

The present study is the first to investigate and analyze the rate of non-fermenting bacteria isolates in IAIs, UTIs and RTIs, as well as the rates of MDR and carbapenem resistance (CR) $A$. baumannii and $P$. aeruginosa isolates and their sensitivities to colistin using data collected by SMART China from 2015 to 2017.

\section{MATERIALS AND METHODS}

\section{Non-fermenting Isolates Obtained From 7 Regions of China (2015 - 2017)}

Our hospital's Human Research Ethics Committee accepted the protocols for the present inquiry (Et Number: S-K238) and waived the requirement for consent.

In total, 3,246 non-fermentative bacteria $(2015, n=2,203$; 2016, $n=2,375 ; 2017, n=2,535)$ isolates were collected in 7 distinct regions of China from 21 hospitals (northeast, north, central south, southwest, east Jiangzhe, and east non-Jiangzhe areas) between 2015 and 2017 inclusively. The total number of samples collected yearly from each hospital ranged between 77 and 250. Each sample was identified and dispatched for analysis in Peking Union Medical College Hospital; re-identification employed MALDITOF MS as the main tool. Duplicate isolates from the same patient were not included in the analysis. The detailed collection criteria for isolates from IAI, UTI, and RTI are as follows:

\section{Sample Collection Criteria for IAls}

The isolate must meet the laboratory criteria of "significant pathogen" and be considered the probable causative agent of infection. Only Gram-negative aerobic and facultative anaerobic bacteria from abdominal infection sites such as the appendix, peritoneum, colon, bile, pelvis and pancreas were included and the strains needed to be pathogenic bacteria associated with clinical infections, while Gram-positive and anaerobic bacteria were excluded. The specimens were mainly obtained through surgical procedures, but puncture specimens such as intraperitoneal puncture fluid were also included and different Gram-negative bacteria that were combined in one sample were also accepted. Exclusion criteria were isolates from drainage liquid or drainage bottles, as well as isolates from feces or perianal abscess environmental samples (not a patient source) or cultures for infection control purposes.

\section{Sample Collection Criteria for UTIs}

The isolate must meet the laboratory criteria of a "significant pathogen" and be considered the probable causative agent of infection. The UTI isolates were obtained from clean catch midstream urine, the urinary bladder, kidney, and the prostate gland.

\section{Sample Collection Criteria for RTls}

The isolate must meet the laboratory criteria of a "significant pathogen" and be considered the probable causative agent of infection. Gram-negative aerobic and facultative bacteria cultured from specimens from lower respiratory tract body sites [e.g., sputum, bronchoalveolar lavage (BAL), thoracentesis, bronchial brushing, endotracheal aspirate, and lung biopsy].

\section{Testing of Organism Susceptibility to Antibiotics}

Antimicrobial susceptibility testing was performed according to the Clinical and Laboratory Standards Institute (CLSI) broth 
microdilution method with custom-made dehydrated Trek Diagnostic Systems panels (Thermo Fisher Scientific, Cleveland, United States) between 2015 and 2017. The susceptibility interpretations were based on the clinical breakpoints recommended by the Clinical and Laboratory Standards Institute [CLSI] (2017). The antimicrobial agents colistin, carbapenems (imipenem, meropenem), an aminoglycoside (amikacin), a cephalosporin (cefepime), a quinolone (ciprofloxacin) and piperacillin/azobactam were tested according to the guidelines of the Surgical Infection Society and Infectious Diseases Society of America (Solomkin et al., 2010). Reference strains of E. coli American Type Culture Collection (ATCC) 25922 and $P$. aeruginosa (ATCC 27853) were used as quality controls for each batch of MIC tests. Data were analyzed only when the quality control test results were acceptable. In the present study, a CR strain was defined as resistant to either imipenem or meropenem. MDR was defined as a strain that was not susceptibility to $\geq 3$ of 6 key antibiotics namely: amikacin; ciprofloxacin; cefepime; colistin; imipenem; and piperacillin/tazobactam. These antibiotics were chosen as they are usually employed in the clinic to treat Gram-negative infections.

\section{RESULTS}

\section{Composition of Non-fermenting Bacteria From 2015 - 2017}

From 2015 to 2017, a total of 3,246 non-fermentative bacteria were collected, of which $P$. aeruginosa and A. baumannii were major isolates of non-fermentative bacteria with similar proportions ( 41.3 and $41.9 \%$, respectively), with the majority isolated from RTIs. However, the overall percentages of MDR and $\mathrm{CR}$ in A. baumannii isolates were as high as 80.1 and $78.7 \%$, respectively, which was almost two times that of MDR $P$. aeruginosa (36.2\%) and CR $P$. aeruginosa (38.9\%) rates. A. baumannii, CR and MDR phenotypes were particularly found in abundance in isolates taken from the respiratory tract $(81.0$ and $82.0 \%$ ) and in IAIs (81.0 and $82.6 \%$ ). Similarly, MDR and CR rates of $P$. aeruginosa strains were essentially highest in isolates from RTIs (41.8 and 44.3\%) followed by IAI isolates (29.3 and $36.1 \%$ ) and lowest in UTI isolates (24.2 and 20.2\%). Apart from somewhat lower MDR $P$. aeruginosa and CR $P$. aeruginosa rates in 2015 there was otherwise an almost constant pattern throughout the years 2015-2017 (Table 1).

\section{Susceptibility Analysis of $A$. baumannii and $P$. aeruginosa to 7 Antimicrobial Agents (2015 - 2017)}

The susceptibility rates to carbapenems of A. baumannii isolates were only about $20 \%$ and that of $P$. aeruginosa was $<60 \%$. Susceptibility rates of $P$. aeruginosa isolates were relatively high to amikacin (88.4\%), but the $\mathrm{MIC}_{90}$ values were $>32 \mathrm{mg} / \mathrm{L}$, while A. baumannii isolates exhibited only $33.04 \%$ susceptibility. The susceptibility rates to ciprofloxacin, cefepime and piperacillin tazobactam were higher in P. aeruginosa isolates (55 - 63\%) than in A. baumannii isolates ( $<20 \%)$. The susceptibility rates to
TABLE 1 | The overall distribution of non-fermentative bacteria, MDR non-fermentative bacteria and CR non-fermentative bacteria collected in 2015, 2016, and 2017

\begin{tabular}{|c|c|c|c|}
\hline 2015-2017 & $\begin{array}{c}\text { No. isolated } \\
\text { N (\%) }\end{array}$ & $\begin{array}{l}\text { MDR } \\
\text { N (\%) }\end{array}$ & $\begin{array}{c}\text { CR } \\
\mathrm{N}(\%)\end{array}$ \\
\hline Non-fermentative bacteria & $3246(100.0)$ & $1754(54.0)$ & 1699 (52.3) \\
\hline A. baumannii & 1360 (41.9) & $1090(80.1)$ & 1070 (78.7) \\
\hline $\mid \mathrm{Al}$ & $327(24.0)$ & $270(82.6)$ & $265(81.0)$ \\
\hline UTI & $96(7.1)$ & $51(53.1)$ & 45 (46.9) \\
\hline RTI & 933 (68.6) & 765 (82.0) & 756 (81.0) \\
\hline$P$. aeruginosa & $1341(41.3)$ & $486(36.2)$ & 522 (38.9) \\
\hline $\mid \mathrm{Al}$ & $321(23.9)$ & $94(29.3)$ & $116(36.1)$ \\
\hline UTI & 198 (14.8) & $48(24.2)$ & $40(20.2)$ \\
\hline $\mathrm{RTI}$ & $815(60.8)$ & $341(41.8)$ & 361 (44.3) \\
\hline Other non-fermentative bacteria & $545(16.8)$ & $178(32.7)$ & $107(19.6)$ \\
\hline \multicolumn{4}{|l|}{2015} \\
\hline Non-fermentative bacteria & 742 & $380(51.2)$ & 363 (48.9) \\
\hline A. baumannii & $283(38.1)$ & $230(81.3)$ & $224(79.2)$ \\
\hline$|A|$ & 115 (40.6) & 98 (85.2) & 97 (84.3) \\
\hline UTI & $25(8.8)$ & $14(56.0)$ & $10(40.0)$ \\
\hline $\mathrm{RTI}$ & $141(49.8)$ & 116(82.3) & $115(81.6)$ \\
\hline$P$. aeruginosa & 320 (43.1) & 88 (27.5) & $104(32.5)$ \\
\hline $\mid \mathrm{Al}$ & $118(36.9)$ & $32(27.1)$ & $42(35.6)$ \\
\hline UTI & 53 (16.6) & $4(7.5)$ & $4(7.5)$ \\
\hline RTI & 149 (46.6) & $52(34.9)$ & 58 (38.9) \\
\hline Other non-fermentative bacteria & 139 (18.7) & $62(44.6)$ & 35 (25.2) \\
\hline
\end{tabular}

Non-fermentative bacteria

A. baumannii

IAI

UTI

$\mathrm{RTI}$

$P$. aeruginosa

IAl

UTI

$\mathrm{RTI}$

\begin{tabular}{|c|c|c|}
\hline 742 & $380(51.2)$ & 363 (48.9) \\
\hline $283(38.1)$ & 230 (81.3) & $224(79.2)$ \\
\hline 115 (40.6) & 98 (85.2) & 97 (84.3) \\
\hline $25(8.8)$ & 14(56.0) & $10(40.0)$ \\
\hline $141(49.8)$ & 116(82.3) & 115 (81.6) \\
\hline 320 (43.1) & 88 (27.5) & 104 (32.5) \\
\hline 118 (36.9) & $32(27.1)$ & 42 (35.6) \\
\hline 53 (16.6) & $4(7.5)$ & $4(7.5)$ \\
\hline 149 (46.6) & $52(34.9)$ & 58 (38.9) \\
\hline 139 (18.7) & $62(44.6)$ & $35(25.2)$ \\
\hline 1091 & $616(56.5)$ & 599 (54.9) \\
\hline 492 (45.1) & 393 (79.9) & $384(78.0)$ \\
\hline 111 (22.6) & 91 (82.0) & $87(78.4)$ \\
\hline 37 (7.5) & $19(51.4)$ & 17 (45.9) \\
\hline 343 (69.7) & 282 (82.2) & 279 (81.3) \\
\hline 449 (41.2) & 176 (39.2) & $184(41.0)$ \\
\hline $108(24.1)$ & 37 (34.3) & $44(40.7)$ \\
\hline $46(10.2)$ & 11 (23.9) & $10(21.7)$ \\
\hline 290 (64.6) & $125(43.1)$ & $125(43.1)$ \\
\hline $150(13.7)$ & 47 (31.3) & 31 (20.7) \\
\hline 1413 & 758 (53.6) & $737(52.2)$ \\
\hline 585 (41.4) & 467 (79.8) & $462(79.0)$ \\
\hline 101 (17.3) & 81 (80.2) & $81(80.2)$ \\
\hline $34(5.8)$ & 18 (52.9) & 18 (52.9) \\
\hline 449 (76.8) & 367 (81.7) & 362 (80.6) \\
\hline 572 (40.5) & 222 (38.8) & 234 (40.9) \\
\hline 95 (16.6) & 25 (26.3) & 30 (31.6) \\
\hline 99 (17.3) & 33 (33.3) & 26 (26.3) \\
\hline 376 (65.7) & 164 (43.6) & 178 (47.3) \\
\hline $256(18.1)$ & 69 (27.0) & 41 (16.0) \\
\hline
\end{tabular}

Other non-fermentative bacteria

2017

Non-fermentative bacteria

A. baumannii

IAI

UTI

$\mathrm{RTI}$

$P$. aeruginosa

IAl

UTI

$\mathrm{RTI}$

Other non-fermentative bacteria

Other non-fermentative bacteria include: Acinetobacter junii; Aeromonas hydrophila; Aeromonas caviae; Acinetobacter Iwoffii; Alcaligenes faecalis; Alcaligenes xylosoxidans; Alcaligenes xylosoxidans ssp.; Morganella morganii; Pseudomonas putida; Stenotrophomonas maltophilia; Flavobacterium meningosepticum; Burkholderia cepacia; Pseudomonas fluorescens.

colistin of both $P$. aeruginosa and A. baumannii were $>90 \%$ (94.6 and $95.7 \%$, respectively) and both $\mathrm{MIC}_{50} / \mathrm{MIC}_{90}$ values were $\leq 1 \mathrm{mg} / \mathrm{L}$ (Table 2). The susceptibilities of both $P$. aeruginosa and 
TABLE 2 | Susceptibility rates of $A$. baumannii and $P$. aeruginosa to 7 antimicrobial agents.

\begin{tabular}{|c|c|c|c|c|c|c|c|}
\hline \multirow[t]{2}{*}{ Organism/antimicrobial agent } & \multirow[t]{2}{*}{$N$} & \multicolumn{3}{|c|}{ MIC (mg/L) } & \multicolumn{3}{|c|}{ MIC interpretation } \\
\hline & & $\mathrm{MIC}_{50}$ & $\mathrm{MIC}_{90}$ & MIC range & Susceptible (\%) & Intermediate (\%) & Resistant (\%) \\
\hline \multicolumn{8}{|l|}{ A. baumannii } \\
\hline Colistin & 1356 & $\leq 1$ & $\leq 1$ & $\leq 1->8$ & 95.7 & 0 & 4.4 \\
\hline Imipenem & 1356 & 32 & $>32$ & $\leq 0.5->32$ & 21.1 & 0.7 & 78.2 \\
\hline Meropenem & 1075 & $>16$ & $>16$ & $\leq 0.12->16$ & 21.3 & 0.7 & 78.0 \\
\hline Amikacin & 1356 & $>32$ & $>32$ & $\leq 4->32$ & 33.0 & 0.7 & 66.3 \\
\hline Ciprofloxacin & 1356 & $>2$ & $>2$ & $\leq 0.25->2$ & 18.4 & 0.6 & 81.0 \\
\hline Cefepime & 1356 & $>32$ & $>32$ & $\leq 1->32$ & 19.2 & 2.3 & 78.5 \\
\hline Piperacillin Tazobactam & 1356 & $>64$ & $>64$ & $\leq 2->64$ & 19.6 & 1.2 & 79.2 \\
\hline \multicolumn{8}{|l|}{$P$. aeruginosa } \\
\hline Colistin & 1341 & $\leq 1$ & 2 & $\leq 1->8$ & 94.6 & 0 & 5.4 \\
\hline Imipenem & 1341 & 2 & 32 & $\leq 0.5->32$ & 56.5 & 6.0 & 37.5 \\
\hline Meropenem & 1021 & 1 & $>16$ & $\leq 0.12->16$ & 58.5 & 6.5 & 35.1 \\
\hline Amikacin & 1341 & $\leq 4$ & $>32$ & $\leq 4->32$ & 88.4 & 0.8 & 10.8 \\
\hline Ciprofloxacin & 1341 & 0.5 & $>2$ & $\leq 0.25->2$ & 63.1 & 6.0 & 31.0 \\
\hline Cefepime & 1341 & 8 & $>32$ & $\leq 1->32$ & 63.1 & 11.1 & 25.8 \\
\hline Piperacillin Tazobactam & 1341 & 16 & $>64$ & $\leq 2->64$ & 55.6 & 13.3 & 31.1 \\
\hline
\end{tabular}

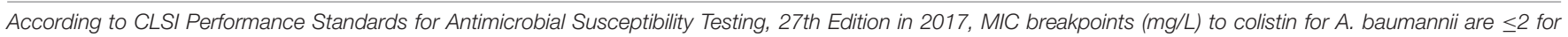
susceptibility and $>2$ for resistance, while MIC breakpoints $(\mathrm{mg} / \mathrm{L})$ to colistin for $P$. aeruginosa are $\leq 2$ for susceptibility and $\geq 4$ for resistance.

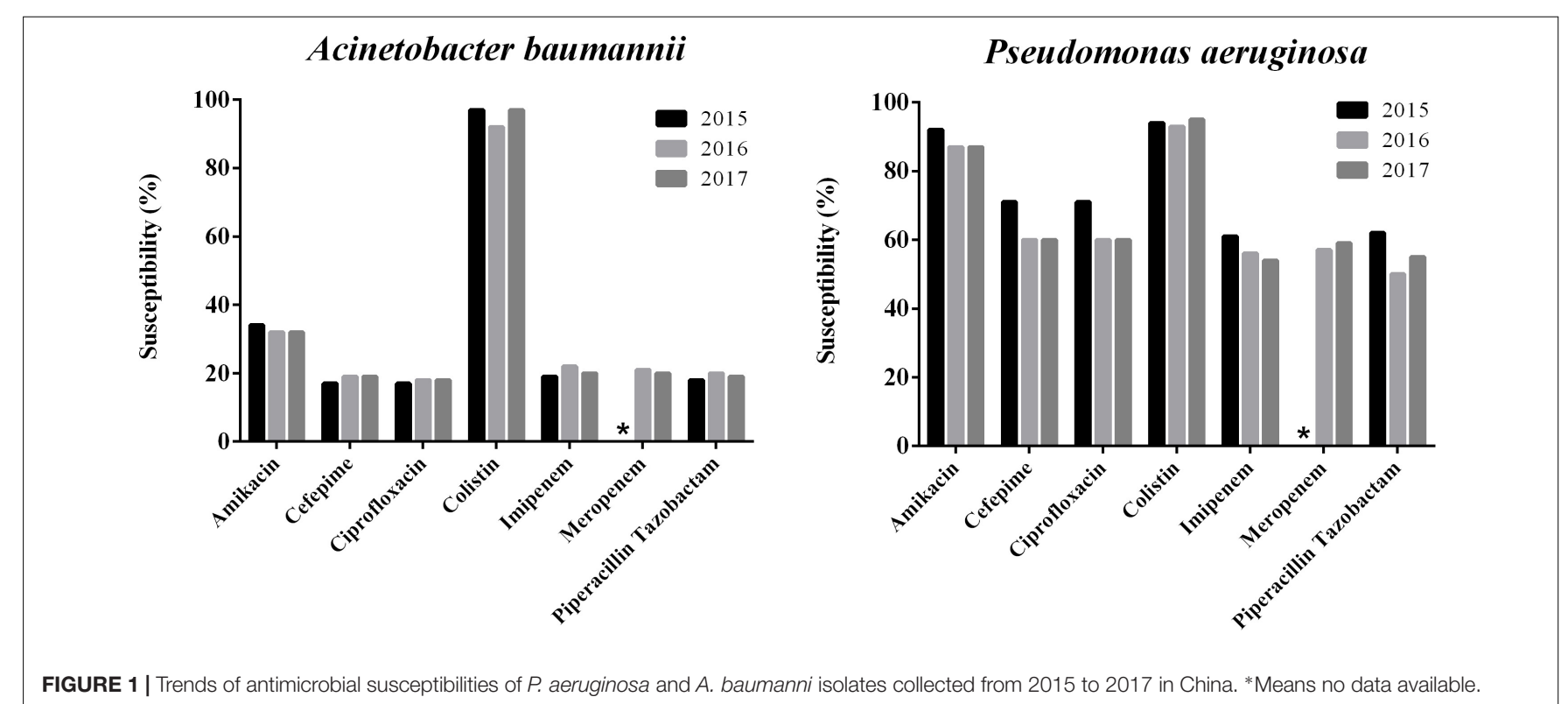

A. baumannii to colistin has been changing in the shape of a " $U$ " curve over the 3 years of the study (Figure 1).

\section{Susceptibility Analysis of MDR}

\section{A. baumannii, CR A. baumannii, MDR $P$. aeruginosa and CR $P$. aeruginosa to Colistin for Different Infection Types From 2015 to 2017}

Colistin (96.1 and 95.4\%) demonstrated highest susceptibility in CR A. baumannii and MDR A. baumannii isolates while susceptibilities to the other antimicrobial agents, with the exception of amikacin (16.8\%), including imipenem as well as ciprofloxacin were $<3 \%$. Colistin susceptibility rates of $\mathrm{CR}$ and MDR A. baumannii isolates were 95.4 and $95.4 \%$ for respiratory tract 98.1 and $97.0 \%$ for IAIs, as well as 95.6 and $92.2 \%$ for UTIs. Susceptibility rates of CR and MDR A. baumannii isolates to amikacin were in the range of $17-18 \%$ for RTI, $14-15 \%$ for IAIs and $7-9 \%$ for UTIs, whereas with the exception of MDR A. baumannii from UTIs with susceptibility rates of $2-12 \%$, all CR and MDR A. baumannii isolates from RTIs, IAIs and UTIs exhibited $<3 \%$ susceptibilities to all other tested antimicrobial agents (Table 3). 
TABLE 3 | In vitro activity of 7 antimicrobial agents against CR and MDR A. baumannii collected in China from 2015 to 2017.

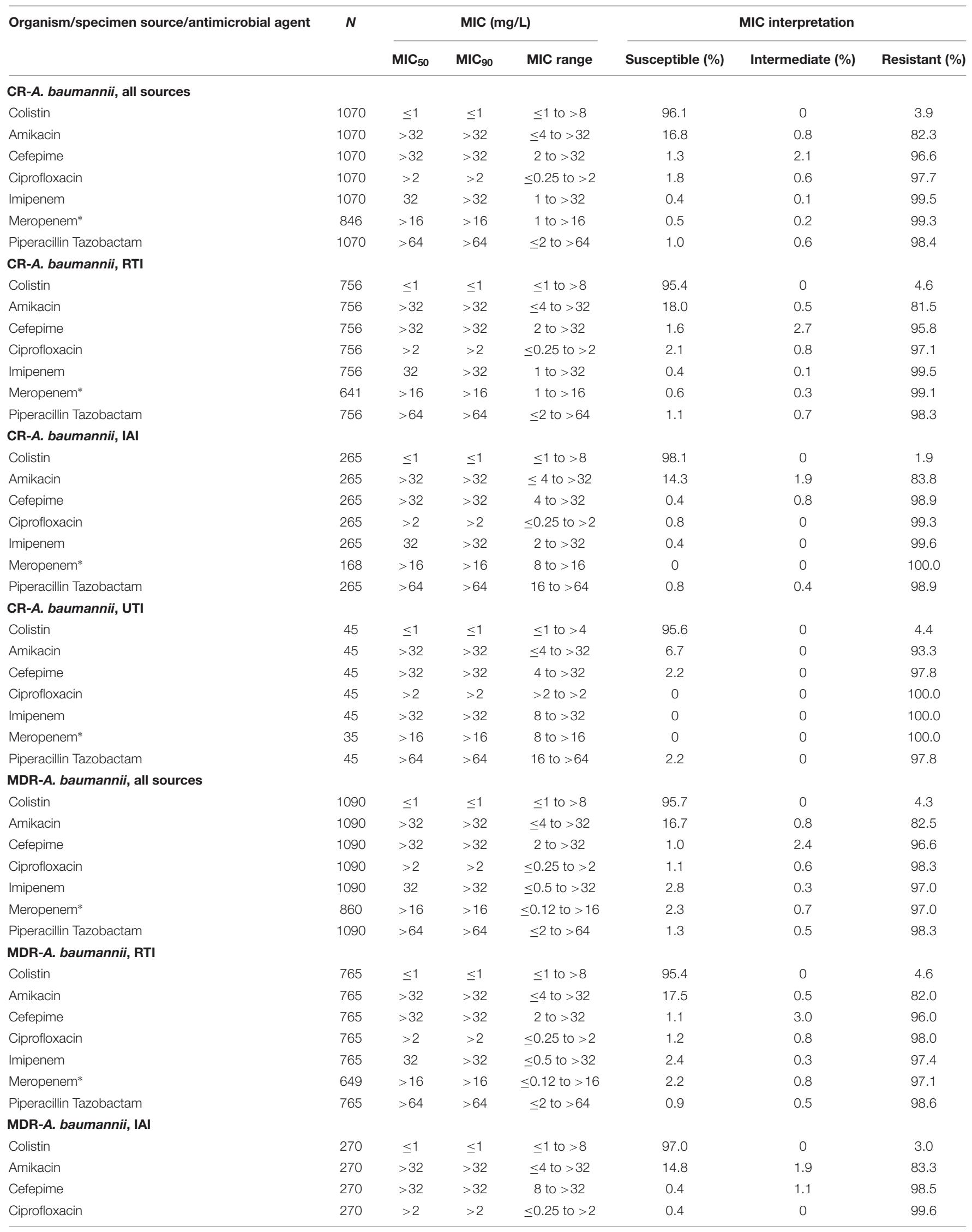


TABLE 3 | Continued

\begin{tabular}{|c|c|c|c|c|c|c|c|}
\hline \multirow[t]{2}{*}{ Organism/specimen source/antimicrobial agent } & \multirow[t]{2}{*}{$N$} & \multicolumn{3}{|c|}{ MIC (mg/L) } & \multicolumn{3}{|c|}{ MIC interpretation } \\
\hline & & $\mathrm{MIC}_{50}$ & $\mathrm{MIC}_{90}$ & MIC range & Susceptible (\%) & Intermediate (\%) & Resistant (\%) \\
\hline Imipenem & 270 & 32 & $>32$ & $\leq 0.5$ to $>32$ & 2.2 & 0.4 & 97.4 \\
\hline Meropenem* & 172 & $>16$ & $>16$ & $\leq 0.12$ to $>16$ & 2.3 & 0.6 & 97.1 \\
\hline Piperacillin Tazobactam & 270 & $>64$ & $>64$ & $\leq 2$ to $>64$ & 1.1 & 0.4 & 98.5 \\
\hline \multicolumn{8}{|l|}{ MDR-A. baumannii, UTI } \\
\hline Colistin & 51 & $\leq 1$ & $\leq 1$ & $\leq 1$ to $>4$ & 92.2 & 0 & 7.8 \\
\hline Amikacin & 51 & $>32$ & $>32$ & $\leq 4$ to $>32$ & 9.8 & 0 & 90.2 \\
\hline Cefepime & 51 & $>32$ & $>32$ & 4 to $>32$ & 3.9 & 0 & 96.1 \\
\hline Ciprofloxacin & 51 & $>2$ & $>2$ & 1 to $>2$ & 2.0 & 2.0 & 96.1 \\
\hline Imipenem & 51 & 32 & $>32$ & $\leq 0.5$ to $>32$ & 11.8 & 0 & 88.2 \\
\hline Meropenem* & 37 & $>16$ & $>16$ & $\leq 0.12$ to $>16$ & 5.4 & 0 & 94.6 \\
\hline Piperacillin Tazobactam & 51 & $>64$ & $>64$ & 4 to $>64$ & 7.8 & 0 & 92.2 \\
\hline
\end{tabular}

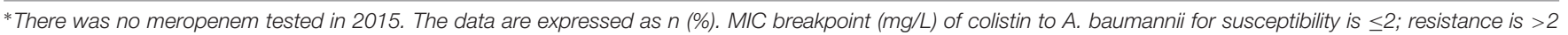
according to Clinical and Laboratory Standards Institute [CLSI] (2017) Performance Standards for Antimicrobial Susceptibility Testing, 27 th Edition, 2017.

Colistin (94.1 and 90.7\%) had the highest susceptibility rates against $\mathrm{CR}$ and MDR $P$. aeruginosa, followed by amikacin (73.4 and 68.7\%). Colistin susceptibility rates of CR and MDR $P$. aeruginosa isolates were 95.6 and $91.79 \%$ for respiratory tract, 89.7 and $87.2 \%$ for intra-abdominal as well as 92.5 and $89.6 \%$ for urinary tract infections. Susceptibilities of CR $P$. aeruginosa to cefepime and ciprofloxacin were $32.7 \%$ for RTIs and in the range of $46.6-53.5 \%$ for IAIs and $20-27.5 \%$ for UTIs, whereas for MDR and CR $P$. aeruginosa RTI, IAI and UTI isolates susceptibilities to the other tested antibiotics were $\leq 35 \%$ (Table 4).

\section{Difference in Sensitivity of $P$. aeruginosa and $A$. baumannii to Colistin in Different Departments}

Overall, sensitivity rates of $P$. aeruginosa and $A$. baumannii to colistin were $>85 \%$ in all departments. There was no pattern of increased resistance rates over the study period in any department and the susceptibilities in 2017 were mostly higher or equal to the rates in 2015 and 2016 in all included departments (Figure 2).

\section{Difference in Sensitivity of $P$. aeruginosa and $A$. baumannii to Colistin in Different Regions}

Similarly to departments, in all regions of China tested, A. baumannii and $P$. aeruginosa sensitivities to colistin were constantly above $80 \%$ and apart from a slight tendency of $P$. aeruginosa and $A$. baumannii in east Jiangzhe there was no visible sensitivity decrease over the years in all other regions (Figure 3).

\section{DISCUSSION}

MDR has become a worldwide problem (WHO, 2014). Since the rise of extended spectrum $\beta$-lactamase-positive
Enterobacteriaceae, carbapenems have served as the gold standard for empirical treatment of ESBL-producing Enterobacteriaceae infections over decades (Papp-Wallace et al., 2011; Vardakas et al., 2012; Tamma et al., 2015). However, since carbapemase-producing Enterobacteriaceae have become increasingly prevalent in the last decade, susceptibility rates, particularly for hospital acquired infections, dropped to levels which urge the need for alternative treatments (Routsi et al., 2013; Zurawski, 2014; Sheu et al., 2018).

In recent years, A. baumannii isolates have developed resistances to carbapenems, amikacin, ciprofloxacin, cefepime and piperacillin-tazobactam (Labarca et al., 2016; Ren et al., 2019). It is noteworthy that CR A. baumannii and CR $P$. aeruginosa are listed as priority pathogens posing the greatest threat to human health by the WHO (Willyard, 2017). In a survey including 312,075 Gram-negative isolates collected in the US in 2017, A. baumannii and P. aeruginosa comprised the most common CR isolates mainly from ICU settings, with rates of 36.6 and 14.6\% (Mccann et al., 2018), which was essentially lower than CR rates of $78.7 \%$ for A. baumannii and $38.9 \%$ for $P$. aeruginosa in the present study, indicating that the situation in China is by far more dramatic compared to the US. However, in our study $P$. aeruginosa and $A$. baumannii isolates were predominantly obtained from RTIs in which the CR and MDR rates of both strains were essentially highest, in good agreement with previously published findings (Mccann et al., 2018). These results may be attributed to the fact that $P$. aeruginosa and $A$. baumannii predominantly cause ventilator-associated pneumonia especially in critically ill patients in ICUs in which CR and MDR rates are generally high (Shete et al., 2010; Tao et al., 2011; Ding et al., 2016; Borgatta et al., 2017; Gupta et al., 2017; Du et al., 2019). However, airborne transmission has also been postulated for the transmission of A. baumannii pneumonia (Whitman et al., 2008; Munoz-Price et al., 2013; Spellberg and Bonomo, 2013).

Susceptibilities of A. baumannii isolates to amikacin, ciprofloxacin, cefepime and piperacillin-tazobactam were 
TABLE 4 | In vitro activity of 7 antimicrobial agents against CR and MDR isolates of P. aeruginosa collected in China from 2015 to 2017.

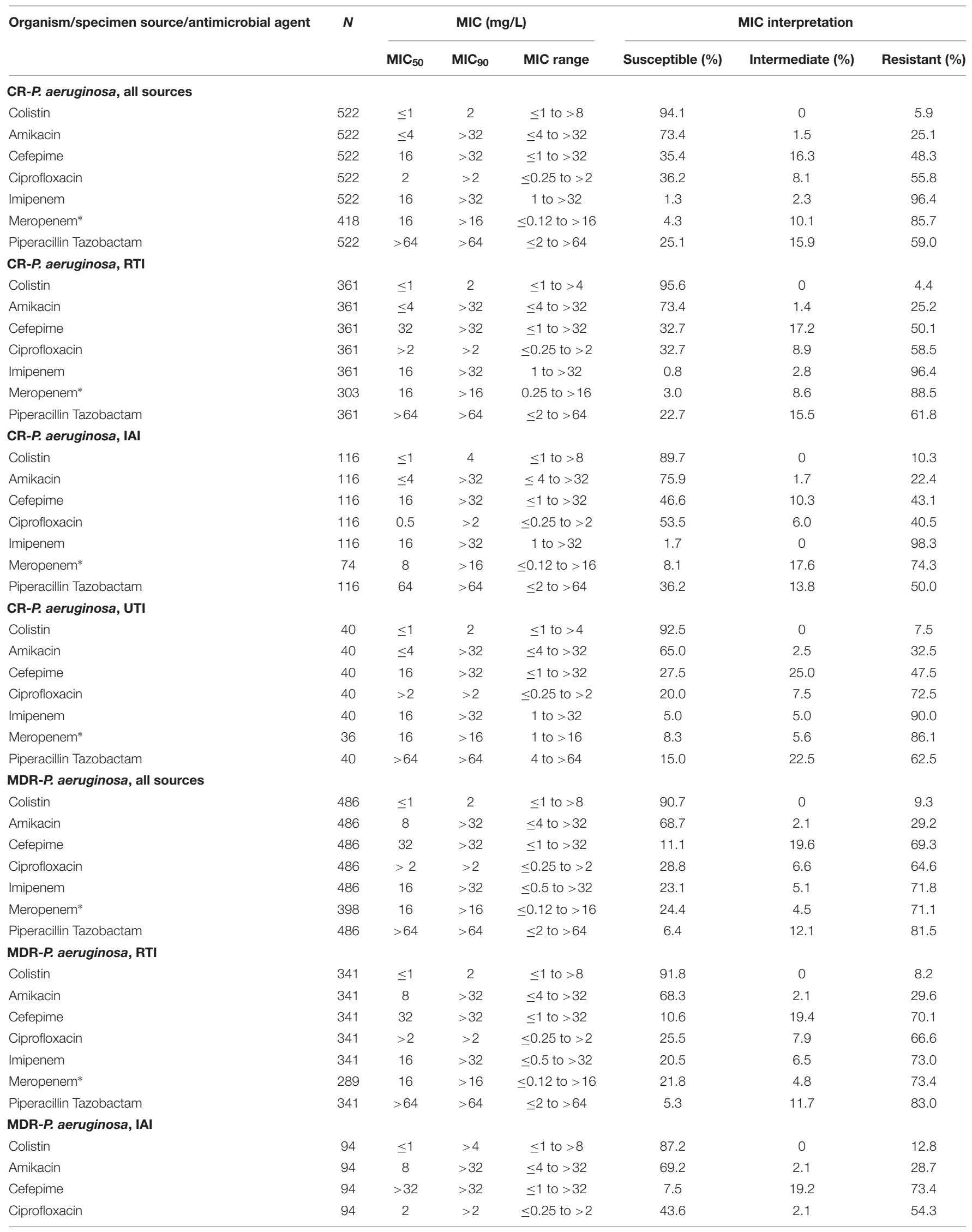


TABLE 4 | Continued

\begin{tabular}{|c|c|c|c|c|c|c|c|}
\hline \multirow[t]{2}{*}{ Organism/specimen source/antimicrobial agent } & \multirow[t]{2}{*}{$N$} & \multicolumn{3}{|c|}{ MIC (mg/L) } & \multicolumn{3}{|c|}{ MIC interpretation } \\
\hline & & $\mathrm{MIC}_{50}$ & $\mathrm{MIC}_{90}$ & MIC range & Susceptible (\%) & Intermediate (\%) & Resistant (\%) \\
\hline Imipenem & 94 & 16 & $>32$ & $\leq 0.5$ to $>32$ & 27.7 & 1.1 & 71.3 \\
\hline Meropenem* & 62 & 8 & $>16$ & $\leq 0.12$ to $>16$ & 30.7 & 4.8 & 64.5 \\
\hline Piperacillin Tazobactam & 94 & $>64$ & $>64$ & $\leq 2$ to $>64$ & 9.6 & 8.5 & 81.9 \\
\hline \multicolumn{8}{|l|}{ MDR-P. aeruginosa, UTI } \\
\hline Colistin & 48 & $\leq 1$ & 4 & $\leq 1$ to $>4$ & 89.6 & 0 & 10.4 \\
\hline Amikacin & 48 & $\leq 4$ & $>32$ & $\leq 4$ to $>32$ & 70.8 & 2.1 & 27.1 \\
\hline Cefepime & 48 & 32 & $>32$ & 4 to $>32$ & 22.9 & 20.8 & 56.3 \\
\hline Ciprofloxacin & 48 & $>2$ & $>2$ & $\leq 0.25$ to $>2$ & 25.0 & 6.3 & 68.8 \\
\hline Imipenem & 48 & 8 & $>32$ & $\leq 0.5$ to $>32$ & 33.3 & 4.2 & 62.5 \\
\hline Meropenem* & 44 & 16 & $>16$ & $\leq 0.12$ to $>16$ & 34.1 & 2.3 & 63.6 \\
\hline Piperacillin Tazobactam & 48 & $>64$ & $>64$ & 4 to $>64$ & 8.3 & 20.8 & 70.8 \\
\hline
\end{tabular}

* Meropenem was not examined in 2015, only after 2016. The data are expressed as $n$ (\%). MIC breakpoint (mg/L) of colistin to P. aeruginosa for susceptibility is $\leq 2$; resistance is > 2 according to Clinical and Laboratory Standards Institute [CLSI] (2017) Performance Standards for Antimicrobial Susceptibility Testing, 27 th Edition, 2017.

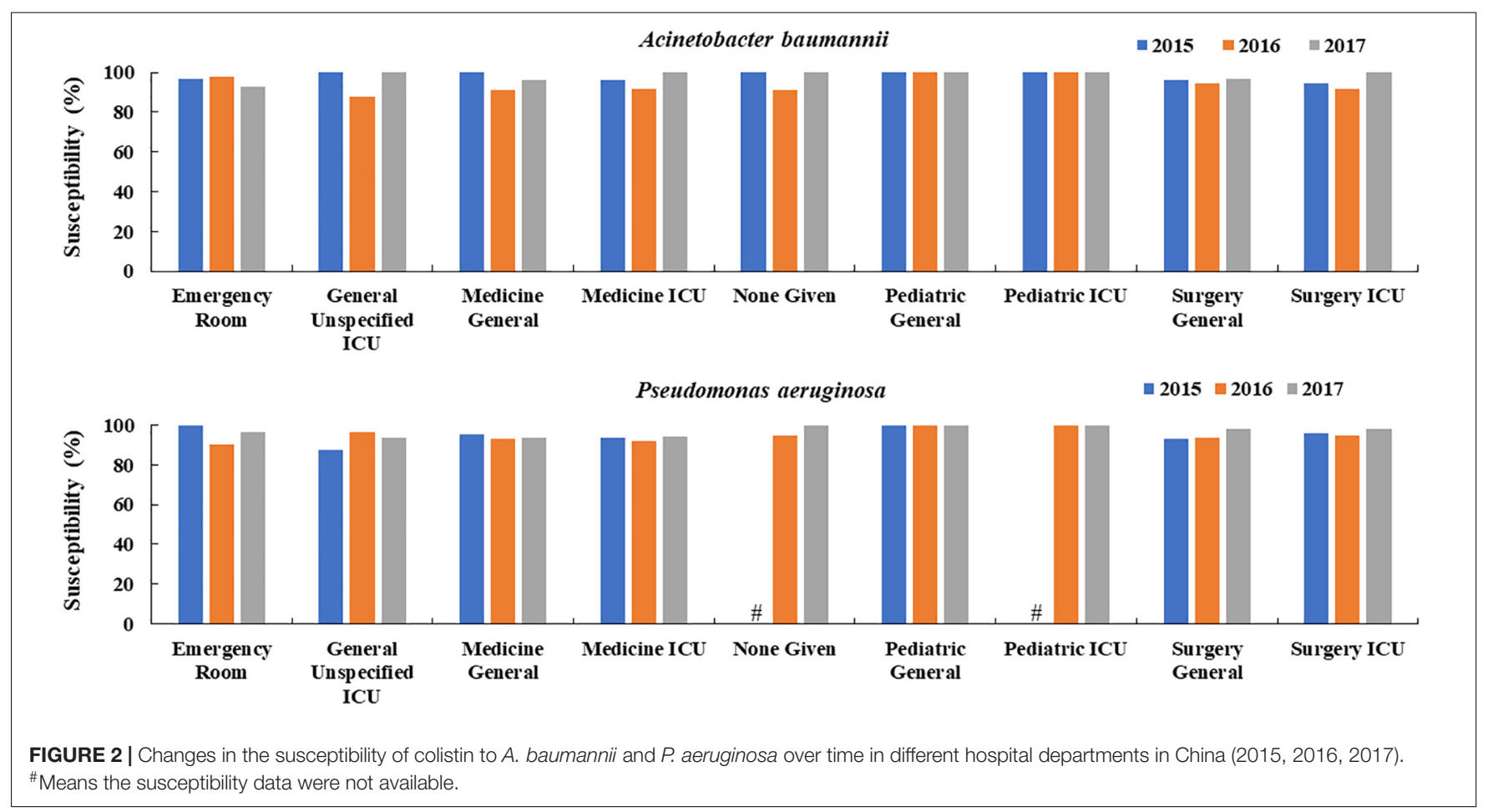

only in the range $19.2-33.0 \%$ and for $P$. aeruginosa 55.6 - $63.1 \%$ to ciprofloxacin, cefepime and piperacillintazobactam, but $83.4 \%$ to amikacin, indicating that for all tested antimicrobial agents other than colistin, at best only amikacin might serve as an empiric treatment option for $P$. aeruginosa infections. In contrast, overall susceptibility rates to colistin were 94.6 and $95.7 \%$ for $P$. aeruginosa and A. baumannii in isolates obtained from IAIs, UTIs and RTIs in the present study, which is in agreement with previously reported high susceptibility rates of both species in other countries (Ece et al., 2014; Malekzadegan et al., 2019). In addition, there was no visible trend of decreasing colistin susceptibilities toward 2017 in the different hospital departments and Chinese regions, except for a slight decrease in east Jiangzhe. The constant low resistant rates might be explained by the fact that colistin has been mainly used for human clinical medication in China since 2017 (WHO, 2018) and earlier colistin resistance of Enterobacteriaceae in China has been attributed to veterinary use (Chen et al., 2011; Yang et al., 2017; Wang et al., 2018). The resistance mechanism to colistin of these small number of isolates may be associated with chromosomal mutations (Dafopoulou et al., 2015) or defective biofilm formation (Azimi and Lari, 2019). 


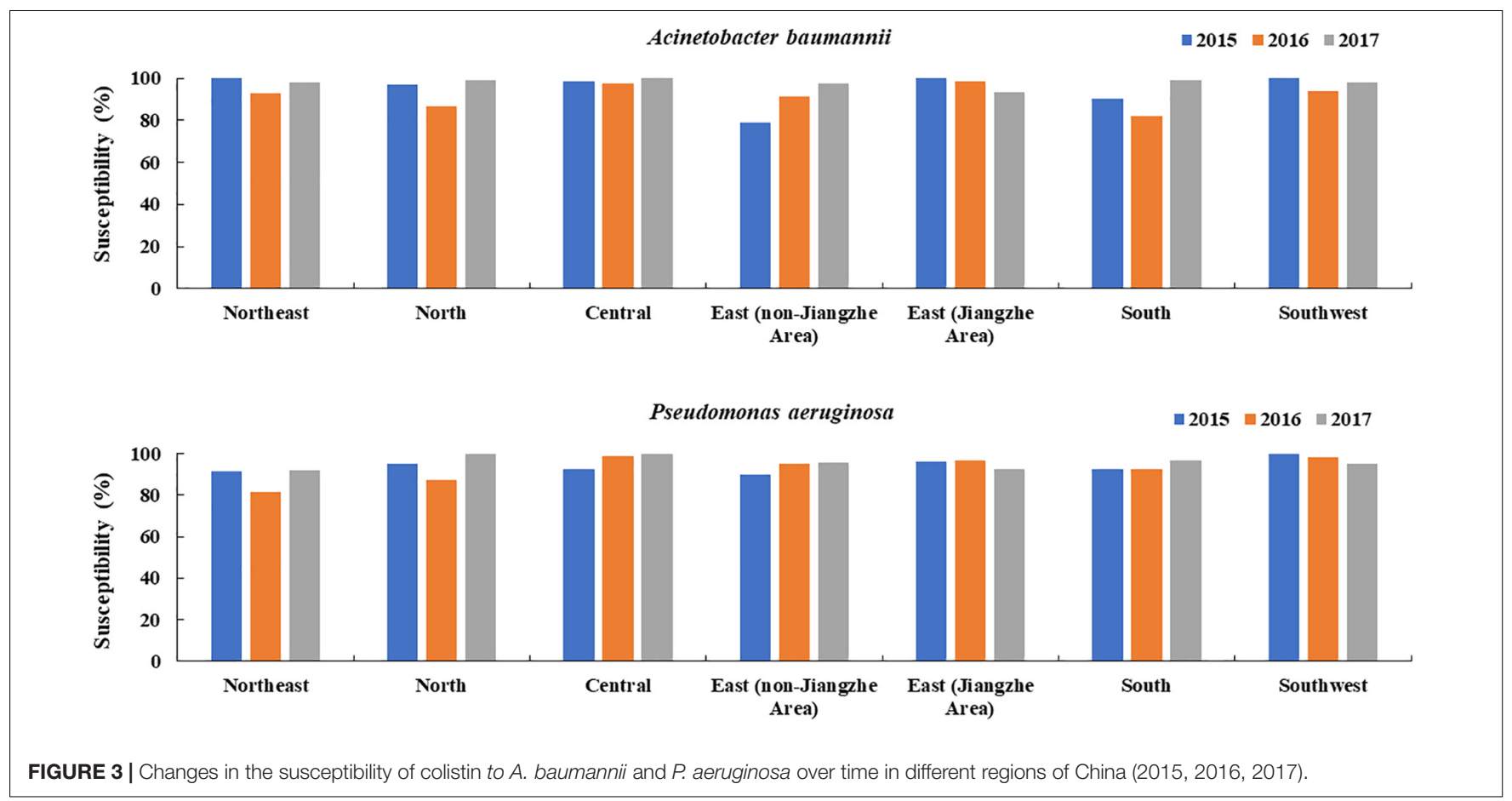

\section{Limitations}

The present study presents only data from 2015 to 2017, because the SMART surveillance program is retrospective. In addition, we reported the susceptibility of $A$. baumannii and $P$. aeruginosa to 7 commonly used antibiotics in China. The susceptibilities of other non-fermenting bacteria such as Stenotrophomonas, Burkholderia were not included due to the limited number of strains.

\section{CONCLUSION}

Due to high MDR and CR rates of A. baumannii (80.1 and 78.7\%) and P. aeruginosa (36.2 and 38.9\%) isolates from RTIs, IAIs and UTIs, between 2015 and 2017 high susceptibility rates were only detected for colistin.

\section{DATA AVAILABILITY STATEMENT}

The SMART database is not public and only accessible for SMART investigators, but the data that support the findings of this study are directly available from MSD China or from the authors upon reasonable request and with permission of MSD China.

\section{ETHICS STATEMENT}

The protocol has been reviewed by the Human Research Ethics Committee of the Institutional Review Board (IRB) of the Peking Union Medical College Hospital and since the project falls under the category observational study and all bacterial strains were from residual samples used in clinical diagnosis or were strains from their subcultures, it met the criteria for exemption. This project did not involve any patient information nor did it affect the normal diagnosis and treatment of patients, and after consultation with the IRB, formal ethical approval was reviewed and waived; written patient consent was not required (Ethics Approval Number: S-K238).

\section{AUTHOR CONTRIBUTIONS}

All the authors read and approved the submitted manuscript and solely responsible for the conception and implementation of the study and for writing the manuscript. HZ, QY, and YX conceptualized the study. HZ, GZ, JZ, SD, YK, QY, and YX collected the data. HZ, QY, and YX analyzed the data. HZ wrote the original draft. HZ, GZ, JZ, SD, YK, QY, and YX wrote, reviewed, and edited the manuscript.

\section{FUNDING}

The authors declare that this study received funding from Merck \& Co., Inc., Kenilworth, NJ, United States. Further support was provided by the National Key Research and Development Program of China (2018YFC1200100 and 2018YFC1200105), the Chinese Academy of Medical Sciences (CAMS) Initiative for Innovative Medicine (Grant No. 2016-I2M-3-014), and the Outstanding Talents Training Funding Project of Dongcheng District, Beijing (2017). The funders were not involved in the study design, collection, analysis, interpretation of data, writing of this article or the decision to submit it for publication. 


\section{ACKNOWLEDGMENTS}

Medical writing and editorial assistance was provided by Shanghai BIOMED Science Technology (Shanghai,

\section{REFERENCES}

Azimi, L., and Lari, A. R. (2019). Colistin-resistant Pseudomonas aeruginosa clinical strains with defective biofilm formation. GMS Hyg. Infect. Control 14, Doc12.

Borgatta, B., Gattarello, S., Mazo, C. A., Imbiscuso, A. T., Larrosa, M. N., Lujàn, M., et al. (2017). The clinical significance of pneumonia in patients with respiratory specimens harbouring multidrug-resistant Pseudomonas aeruginosa: a 5year retrospective study following 5667 patients in four general ICUs. Eur. J. Clin. Microbiol. Infect. Dis. 36, 2155-2163. doi: 10.1007/s10096-0173039-z

Castanheira, M., Deshpande, L. M., Costello, A., Davies, T. A., and Jones, R. N. (2014). Epidemiology and carbapenem resistance mechanisms of carbapenemnon-susceptible Pseudomonas aeruginosa collected during 2009-11 in 14 European and Mediterranean countries. J. Antimicrob. Chemother. 69, 1804 1814. doi: 10.1093/jac/dku048

Chen, B., He, L., Hu, B., Ni, Y., Q, H., S, Y., et al. (2012). Chinese Expert Consensus for the Diagnosis, Treatment, Prevention and Control of Acinetobacter Baumannii. Infect. China Med. Pharm. 92, 76-85.

Chen, S., Hu, F., Zhang, X., Xu, X., Liu, Y., Zhu, D., et al. (2011). Independent emergence of colistin-resistant Enterobacteriaceae clinical isolates without colistin treatment. J. Clin. Microbiol. 49, 4022-4023. doi: 10.1128/jcm.012 33-11

Chopra, T., Marchaim, D., Awali, R. A., Krishna, A., Johnson, P., Tansek, R., et al. (2013). Epidemiology of bloodstream infections caused by Acinetobacter baumannii and impact of drug resistance to both carbapenems and ampicillinsulbactam on clinical outcomes. Antimicrob. Agents Chemother. 57, 6270-6275. doi: 10.1128/aac.01520-13

Clinical and Laboratory Standards Institute [CLSI] (2017). Performance Standards for Antimicrobial Susceptibility Testing, 27th Edn. Annapolis Junction, MD: CLSI.

Dafopoulou, K., Xavier, B. B., Hotterbeekx, A., Janssens, L., Lammens, C., Dé, E., et al. (2015). Colistin-Resistant Acinetobacter baumannii Clinical Strains with Deficient Biofilm Formation. Antimicrob. Agents Chemother. 60, 1892-1895. doi: 10.1128/aac.02518-15

Dimopoulos, G., Akova, M., Rello, J., and Poulakou, G. (2020). Understanding resistance in Pseudomonas. Intensive Care Med. 46, 350-352. doi: 10.1007/ s00134-019-05905-6

Ding, C., Yang, Z., Wang, J., Liu, X., Cao, Y., Pan, Y., et al. (2016). Prevalence of Pseudomonas aeruginosa and antimicrobial-resistant Pseudomonas aeruginosa in patients with pneumonia in mainland China: a systematic review and meta-analysis. Int. J. Infect. Dis. 49, 119-128. doi: 10.1016/j.ijid.2016.06.014

Du, X., Xu, X., Yao, J., Deng, K., Chen, S., Shen, Z., et al. (2019). Predictors of mortality in patients infected with carbapenem-resistant Acinetobacter baumannii: a systematic review and meta-analysis. Am. J. Infect. Control 47, 1140-1145. doi: 10.1016/j.ajic.2019.03.003

Ece, G., Samlioglu, P., Atalay, S., and Kose, S. (2014). Evaluation of the in vitro colistin susceptibility of Pseudomonas aeruginosa and Acinetobacter baumannii strains at a tertiary care centre in Western Turkey. Infez. Med. 22, 36-40.

El Chakhtoura, N. G., Saade, E., Iovleva, A., Yasmin, M., Wilson, B., Perez, F., et al. (2018). Therapies for multidrug resistant and extensively drug-resistant nonfermenting gram-negative bacteria causing nosocomial infections: a perilous journey toward 'molecularly targeted' therapy. Expert Rev. Anti Infect. Ther. 16, 89-110. doi: 10.1080/14787210.2018.1425139

Fernández-Barat, L., Ferrer, M., De Rosa, F., Gabarrús, A., Esperatti, M., Terraneo, S., et al. (2017). Intensive care unit-acquired pneumonia due to Pseudomonas aeruginosa with and without multidrug resistance. J. Infect. 74, 142-152. doi: 10.1016/j.jinf.2016.11.008

Gupta, R., Malik, A., Rizvi, M., Ahmed, M., and Singh, A. (2017). Epidemiology of multidrug-resistant Gram-negative pathogens isolated from ventilatorassociated pneumonia in ICU patients. J. Glob. Antimicrob. Resist. 9, 47-50. doi: 10.1016/j.jgar.2016.12.016
China) with funding provided by MSD China. The authors were solely responsible for the conception and implementation of this study and for writing the manuscript.
Karageorgopoulos, D. E., and Falagas, M. E. (2008). Current control and treatment of multidrug-resistant Acinetobacter baumannii infections. Lancet Infect. Dis. 8, 751-762. doi: 10.1016/s1473-3099(08)70279-2

Koulenti, D., Tsigou, E., and Rello, J. (2017). Nosocomial pneumonia in 27 ICUs in Europe: perspectives from the EU-VAP/CAP study. Eur. J. Clin. Microbiol. Infect. Dis. 36, 1999-2006. doi: 10.1007/s10096-016-2703-Z

Labarca, J. A., Salles, M. J., Seas, C., and Guzman-Blanco, M. (2016). Carbapenem resistance in Pseudomonas aeruginosa and Acinetobacter baumannii in the nosocomial setting in Latin America. Crit. Rev. Microbiol. 42, 276-292.

Magill, S. S., Edwards, J. R., Bamberg, W., Beldavs, Z. G., Dumyati, G., Kainer, M. A., et al. (2014). Multistate point-prevalence survey of health care-associated infections. N. Engl. J. Med. 370, 1198-1208. doi: 10.1056/nejmoa1306801

Malekzadegan, Y., Abdi, A., Heidari, H., Moradi, M., Rastegar, E., and Sedigh Ebrahim-Saraie, H. (2019). In vitro activities of colistin, imipenem and ceftazidime against drug-resistant Pseudomonas aeruginosa and Acinetobacter baumannii isolates in the south of Iran. BMC Res. Notes 12:301. doi: 10.1186/ s13104-019-4344-7

Mccann, E., Srinivasan, A., Deryke, C. A., Ye, G., Depestel, D. D., Murray, J., et al. (2018). Carbapenem-Nonsusceptible Gram-Negative Pathogens in ICU and Non-ICU Settings in US Hospitals in 2017: A Multicenter Study. Open Forum Infect. Dis. 5:ofy241.

Mesaros, N., Nordmann, P., Plésiat, P., Roussel-Delvallez, M., Van Eldere, J., Glupczynski, Y., et al. (2007). Pseudomonas aeruginosa: resistance and therapeutic options at the turn of the new millennium. Clin. Microbiol. Infect. 13, 560-578. doi: 10.1111/j.1469-0691.2007.01681.x

Munoz-Price, L. S., Fajardo-Aquino, Y., Arheart, K. L., Cleary, T., Depascale, D., Pizano, L., et al. (2013). Aerosolization of Acinetobacter baumannii in a trauma ICU. Crit. Care Med. 41, 1915-1918. doi: 10.1097/ccm.0b013e31828a39c0

Pachon, J., and Vila, J. (2009). Treatment of multiresistant Acinetobacter baumannii infections. Curr. Opin. Investig. Drugs 10, 150-156.

Papp-Wallace, K. M., Endimiani, A., Taracila, M. A., and Bonomo, R. A. (2011). Carbapenems: past, present, and future. Antimicrob. Agents Chemother. 55, 4943-4960.

Perez, F., Hujer, A. M., Hujer, K. M., Decker, B. K., Rather, P. N., and Bonomo, R. A. (2007). Global challenge of multidrug-resistant Acinetobacter baumannii. Antimicrob. Agents Chemother. 51, 3471-3484.

Ren, J., Li, X., Wang, L., Liu, M., Zheng, K., and Wang, Y. (2019). Risk Factors and Drug Resistance of the MDR Acinetobacter Baumannii in Pneumonia Patients in ICU. Open Med. 14, 772-777. doi: 10.1515/med-2019-0090

Routsi, C., Pratikaki, M., Platsouka, E., Sotiropoulou, C., Papas, V., Pitsiolis, T., et al. (2013). Risk factors for carbapenem-resistant Gram-negative bacteremia in intensive care unit patients. Intensive Care Med. 39, 1253-1261. doi: 10.1007/ s00134-013-2914- $z$

Ruiz-Garbajosa, P., and Canton, R. (2017). Epidemiology of antibiotic resistance in Pseudomonas aeruginosa. Implications for empiric and definitive therapy. Rev. Esp. Quimioter. 30(Suppl. 1), 8-12.

Shete, V. B., Ghadage, D. P., Muley, V. A., and Bhore, A. V. (2010). Multidrug resistant Acinetobacter ventilator-associated pneumonia. Lung India 27, 217-220.

Sheu, C. C., Lin, S. Y., Chang, Y. T., Lee, C. Y., Chen, Y. H., and Hsueh, P. R. (2018). Management of infections caused by extended-spectrum beta-lactamaseproducing Enterobacteriaceae: current evidence and future prospects. Expert Rev. Anti Infect. Ther. 16, 205-218.

Solomkin, J. S., Mazuski, J. E., Bradley, J. S., Rodvold, K. A., Goldstein, E. J., Baron, E. J., et al. (2010). Diagnosis and management of complicated intra-abdominal infection in adults and children: guidelines by the Surgical Infection Society and the Infectious Diseases Society of America. Clin. Infect. Dis. 50, 133-164. doi: $10.1086 / 649554$

Spellberg, B., and Bonomo, R. A. (2013). "Airborne assault": a new dimension in Acinetobacter baumannii transmission. Crit. Care Med. 41, 2042-2044. doi: $10.1097 / \mathrm{ccm} .0 \mathrm{~b} 013 \mathrm{e} 31829136 \mathrm{c} 3$ 
Tamma, P. D., Han, J. H., Rock, C., Harris, A. D., Lautenbach, E., Hsu, A. J., et al. (2015). Carbapenem therapy is associated with improved survival compared with piperacillin-tazobactam for patients with extended-spectrum $\beta$-lactamase bacteremia. Clin. Infect. Dis. 60, 1319-1325.

Tao, L., Hu, B., Rosenthal, V. D., Gao, X., and He, L. (2011). Device-associated infection rates in 398 intensive care units in Shanghai, China: International Nosocomial Infection Control Consortium (INICC) findings. Int. J. Infect. Dis. 15, e774-e780. doi: 10.1016/j.ijid.2011.06.009

Towner, K. J. (2009). Acinetobacter: an old friend, but a new enemy. J. Hosp. Infect. 73, 355-363. doi: 10.1016/j.jhin.2009.03.032

Vardakas, K. Z., Tansarli, G. S., Rafailidis, P. I., and Falagas, M. E. (2012). Carbapenems versus alternative antibiotics for the treatment of bacteraemia due to Enterobacteriaceae producing extended-spectrum beta-lactamases: a systematic review and meta-analysis. J. Antimicrob. Chemother. 67, 2793-2803. doi: $10.1093 /$ jac/dks301

Wang, X., Wang, Y., Wang, Y., Zhang, S., Shen, Z., and Wang, S. (2018). Emergence of the colistin resistance gene mcr-1 and its variant in several uncommon species of Enterobacteriaceae from commercial poultry farm surrounding environments. Vet. Microbiol. 219, 161-164. doi: 10.1016/j.vetmic.2018. 04.002

Whitman, T. J., Qasba, S. S., Timpone, J. G., Babel, B. S., Kasper, M. R., English, J. F., et al. (2008). Occupational transmission of Acinetobacter baumannii from a United States serviceman wounded in Iraq to a health care worker. Clin. Infect. Dis. 47, 439-443. doi: 10.1086/589247

WHO, (2014). Antimicrobial Resistance: Global Report on Surveillance. Geneva: WHO.
WHO, (2018). The Detection and Reporting of Colistin Resistance. Geneva: World Health Organization.

Willyard, C. (2017). The drug-resistant bacteria that pose the greatest health threats. Nature 543:15. doi: 10.1038/nature.2017.21550

Yang, Y. Q., Li, Y. X., Song, T., Yang, Y. X., Jiang, W., Zhang, A. Y., et al. (2017). Colistin Resistance Gene mcr-1 and Its Variant in Escherichia coli Isolates from Chickens in China. Antimicrob. Agents Chemother. 61:e01204-16.

Yuan, Z., and Tam, V. H. (2008). Polymyxin B: a new strategy for multidrugresistant Gram-negative organisms. Expert Opin. Investig. Drugs 17, 661-668. doi: $10.1517 / 13543784.17 .5 .661$

Zurawski, R. M. (2014). Carbapenem-resistant enterobacteriaceae: occult threat in the intensive care unit. Crit. Care Nurse 34, 44-52.

Conflict of Interest: YK was employed by MSD China.

The remaining authors declare that the research was conducted in the absence of any commercial or financial relationships that could be construed as a potential conflict of interest.

Copyright (c) 2020 Zhang, Zhang, Zhang, Duan, Kang, Yang and Xu. This is an open-access article distributed under the terms of the Creative Commons Attribution License (CC BY). The use, distribution or reproduction in other forums is permitted, provided the original author(s) and the copyright owner(s) are credited and that the original publication in this journal is cited, in accordance with accepted academic practice. No use, distribution or reproduction is permitted which does not comply with these terms. 